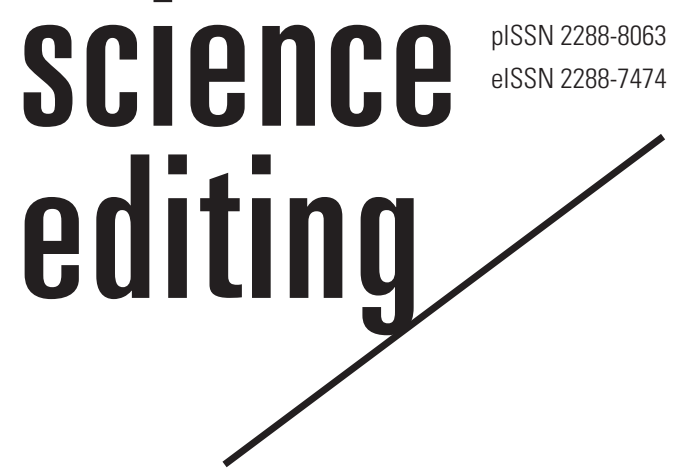

\title{
How to share data through Harvard Dataverse, a repository site: a case of the World Journal of Men's Health
}

\author{
Hyun Jun Park ${ }^{1,2}$ \\ ${ }^{1}$ Department of Urology, Pusan National University School of Medicine, Busan; ${ }^{2}$ Medical Research Institute of Pusan National
} University Hospital, Busan, Korea

Abstract

Data are a highly valuable asset for researchers. Earlier, researchers who conducted a study permanently owned their data. Currently, however, these data can be used as a source for performing further research. In 2018, the International Committee of Medical Journal Editors presented data sharing statements for clinical trials. Although this recommendation was limited to clinical trials published in medical journals, it is a meaningful change that formalized the concept of data sharing. However, the trend of data sharing is expected to spread beyond medical journals to include a wider range of scientific journals in the near future. Correspondingly, platforms that provide storage and services to share data will gradually diversify. The World Journal of Men's Health has adopted a clinical data sharing policy. The data deposit process to Harvard Dataverse, a well-known data repository, is as follows: first, select the type of article for data sharing; second, create an account; third, write a letter to the corresponding author; fourth, receive and validate data from the authors; fifth, upload the data to Harvard Dataverse; and sixth, add a data sharing statement to the paper. It is recommended that scientific journal editors select an appropriate platform and participate in the new trend of data sharing.

Keywords

Data sharing; Statements; Publication; Harvard Dataverse

Received: January 24, 2022

Accepted: January 28, 2022

Correspondence to Hyun Jun Park joon501@pusan.ac.kr

ORCID

Hyun Jun Park

https://orcid.org/0000-0003-0566-9574

\section{Introduction}

It is very rewarding for researchers to inform their colleagues and the public about their research achievements through various channels of communication. The scientific advances achieved by researchers have often led to various studies on similar subjects and follow-up studies, thereby resulting in advances in the corresponding research field. By using data from previous studies, fellow researchers have the opportunity to achieve more advanced results 
through research building upon earlier results. Therefore, research data are regarded as a valuable asset that can be shared by all researchers, including those who conduct the research. In 2018, the International Committee of Medical Journal Editors presented data sharing statements for clinical trials [1]. Although this recommendation was limited to clinical trials published in medical journals, it is a meaningful change that formalized the concept of data sharing. Although many journal websites contain a data sharing policy, few journals actually apply a data sharing policy. Furthermore, several journals have not yet adopted a policy. I would like to introduce the data sharing implemented by the World Journal of Men's Health (WJMH) [1-3]. WJMH uses the Harvard Dataverse repository (https://dataverse.harvard.edu). Although this is just one example of a data sharing method, I hope that it can alleviate some of the technical difficulties that the readers of this article may experience.

\section{How to Deposit Data to the Harvard Dataverse Repository}

Step 1. Select the type of article for data sharing Although the International Committee of Medical Journal Editors recommends sharing data for clinical trials, data shar- ing is possible for any paper, including basic research and meta-analyses, according to the decision of the journal's editorial committee. Therefore, the first step is to select the types of papers to which the data sharing policy is applicable. WJMH currently recommends data sharing for all types of papers, except for narrative reviews and case reports.

\section{Step 2. Create an account}

Access https://dataverse.harvard.edu/ and create an account under the name of the editor-in-chief who is in charge of the journal or editorial office staff. It is important to ensure that the account can be maintained even if the editor changes. The name of the newly created Dataverse account should preferably be the same as the name of the journal (Fig. 1). Currently, the service is free to use and each account is allocated $1 \mathrm{~TB}$ of storage.

Step 3. Write a letter to the corresponding author After a paper submitted to the journal undergoes peer review and is accepted, the editor sends a letter to the corresponding author requesting data to be provided. If the authors do not wish to share the data, they are instructed to state the reason. The letter sent by WJMH provides an option for the authors to indicate their reasons for not sharing the data (Fig. 2). However, a letter to the corresponding author is not necessary

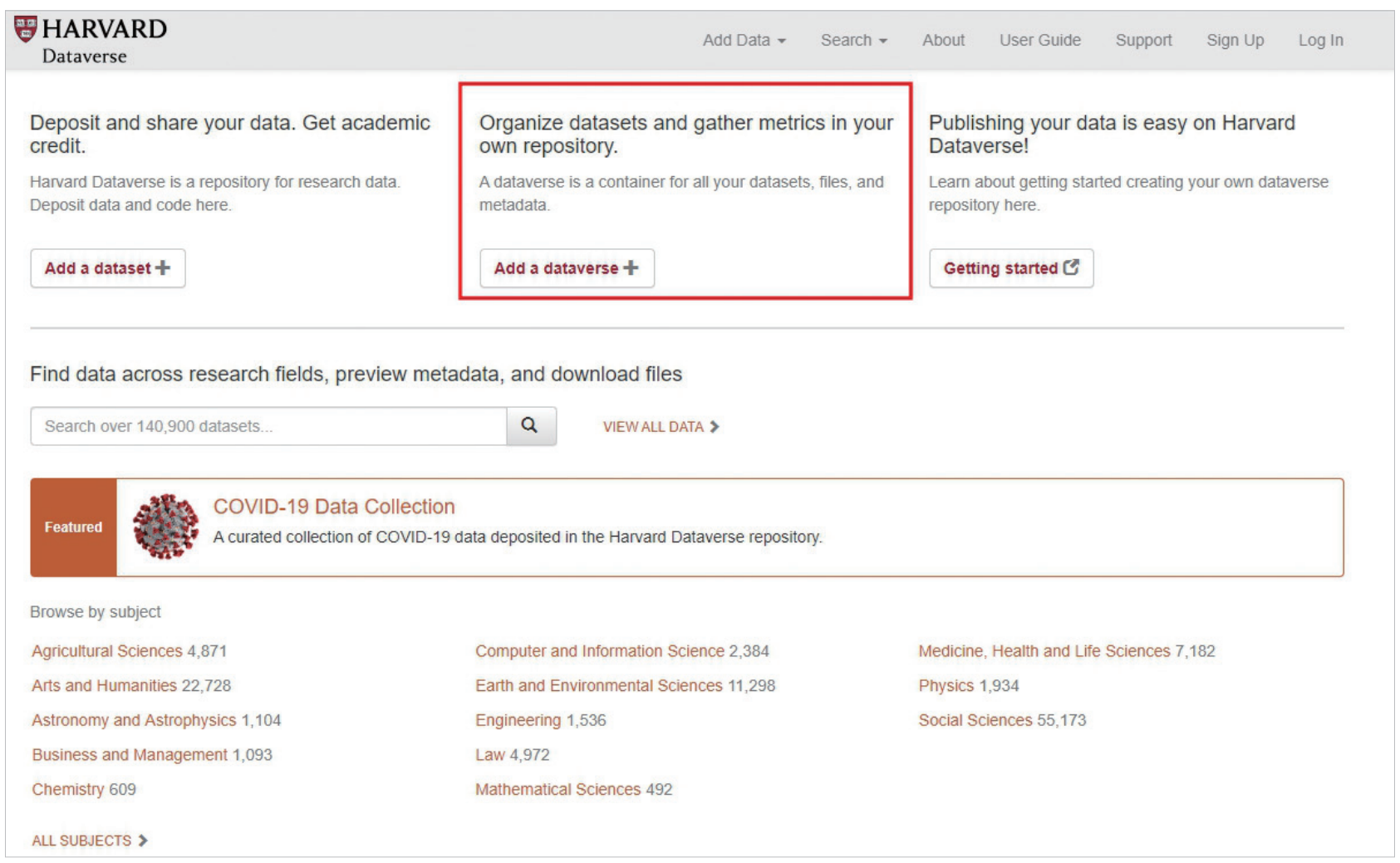

Fig. 1. Creating an account in Harvard Dataverse. 
We are going to upload the data to our space in Harvard Dataverse. So, you don't need to upload the file yourself. If you agree, please just send your data to me via e-mail.

However, if your data cannot be publicized, you can choose one of the reasons below.

I ( ) The data that support the findings of this study are available from the corresponding author upon reasonable request.

$2($ ) The data required to reproduce these findings cannot be shared at this time due to legal and ethical reasons.

3 ( ) The data required to reproduce these findings cannot be shared at this time due to technical and time limitations.

4 ( ) The data required to reproduce these findings cannot be shared at this time as the data also forms part of an ongoing study. .

j.(

5.( ) The data required to reproduce these findings cannot be shared at this time due to personal information protection policy.

$+$

Thank you for your fine contribution.

We are looking forward to your submission to our journal in the future.

$\downarrow$

Best regards. $\downarrow$

Fig. 2. An example of a letter.

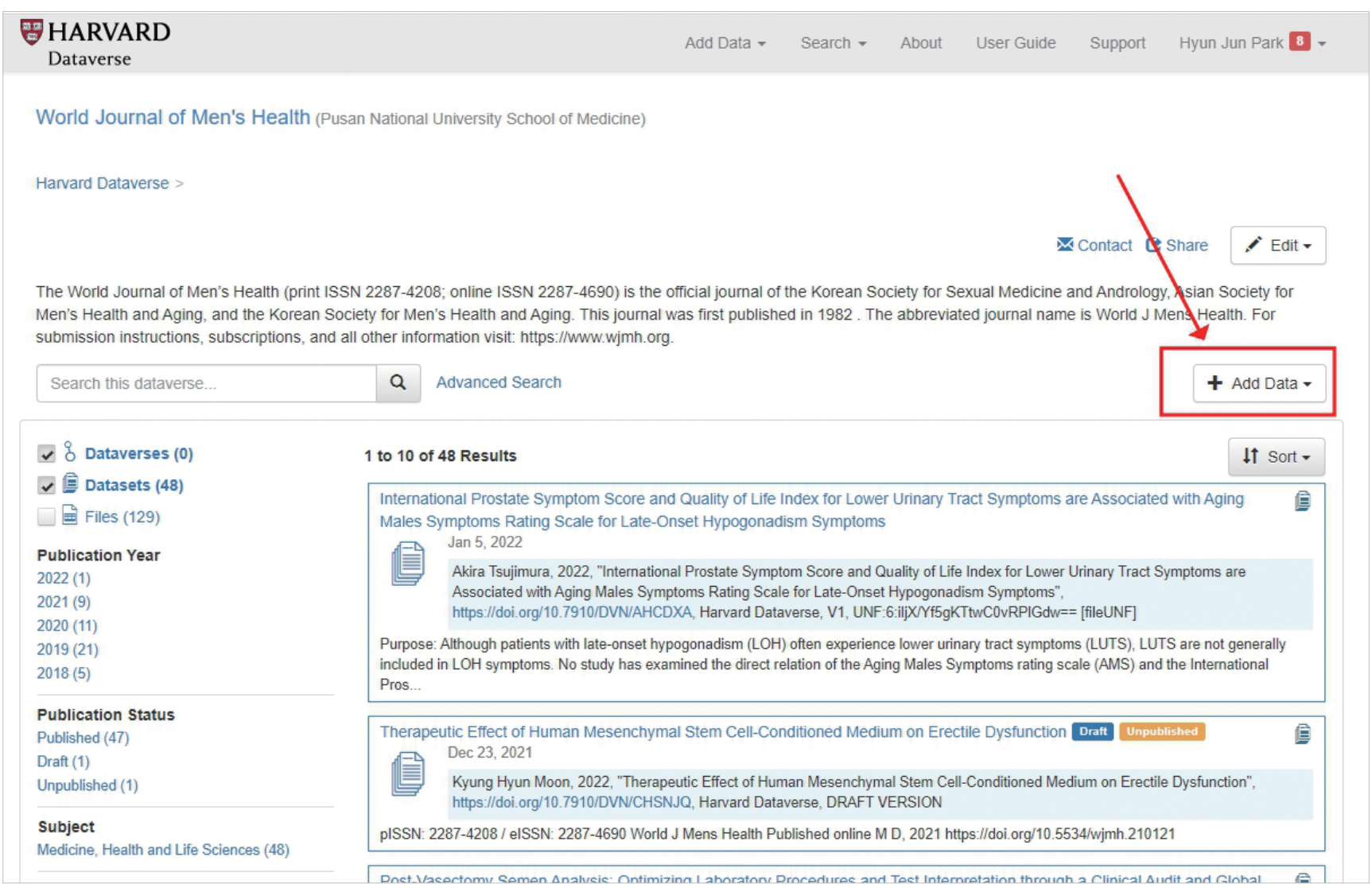

Fig. 3. Adding a new dataset. 


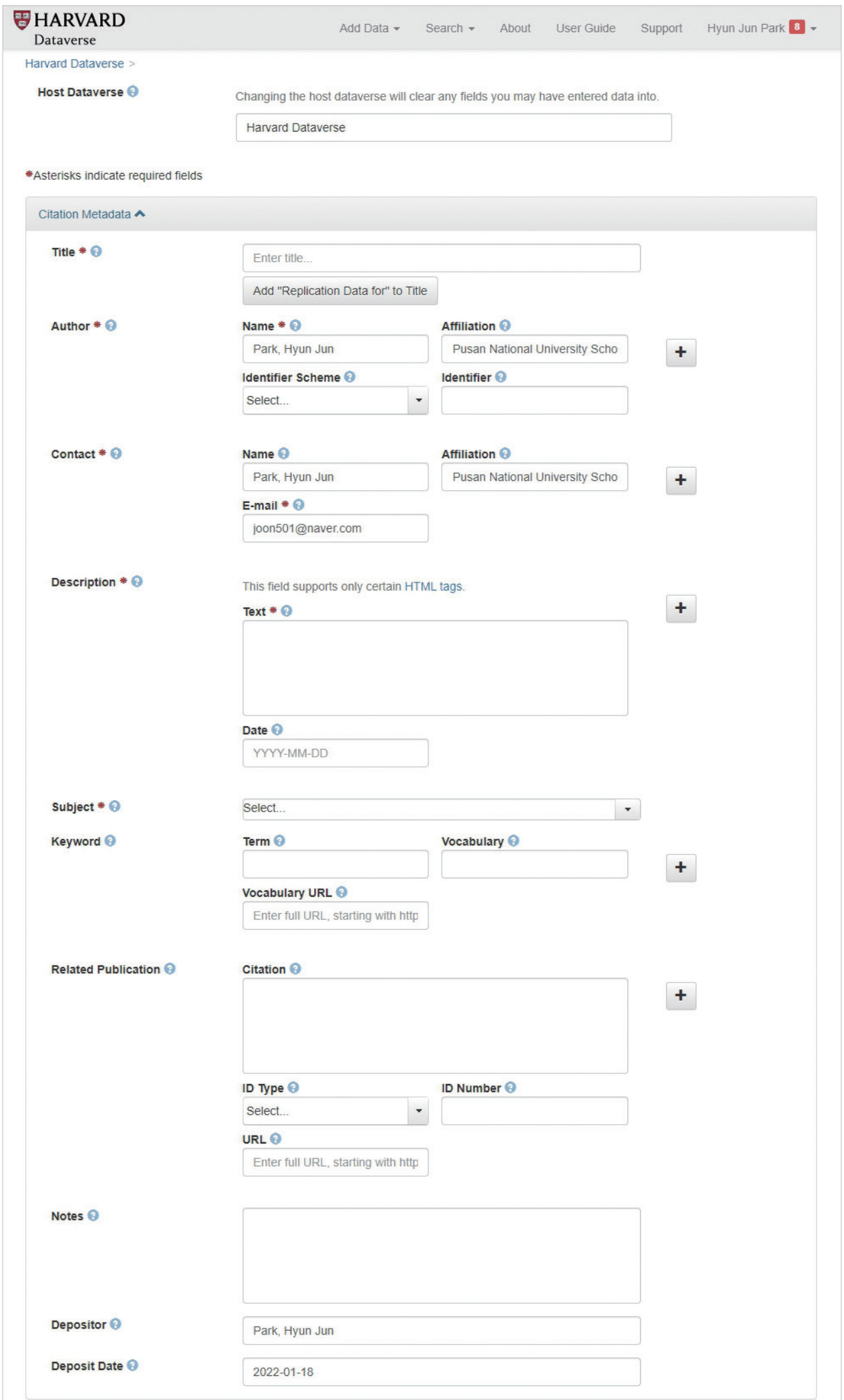

Fig. 4. Filling out the form to upload data. 
if the journal editor receives data during the submission process as part of the journal's mandatory data sharing policy.

Step 4. Receive and validate data from the authors

When data are obtained from the author, they are temporarily stored for each paper in the editorial office. This is the point when data verification is required. Whether personal information is included or exposed is the most important factor to evaluate. Data should be in a format that can be used in statistical programs, unlike texts, graphs, figures, and tables provided as supplements to a paper.

\section{Step 5. Upload the data to Harvard Dataverse}

When the accepted paper is fully edited, the previously received data file is uploaded to Harvard Dataverse. Access the Harvard Dataverse webpage and select 'add data' and 'new da- taset' (Fig. 3). On the screen for uploading data, fill in all of the following items as instructed on the webpage (Fig. 4) [2]. Title: write the title of the article; author, contact: enter the corresponding author's information; description: write a brief summary of the content of the article. The abstract of the article is usually included in this section; subject: choose the field of study; and related publication: enter the article's DOI, expected publication date, journal name, the ISSN (International Standard Serial Number) of the journal, and web page address. When this process is completed, data sharing is completed, and the DOI is assigned to the shared data.

Step 6. Add a data sharing statement to the paper Add the data sharing statement to the final proof of the edited paper (Fig. 5) [4].

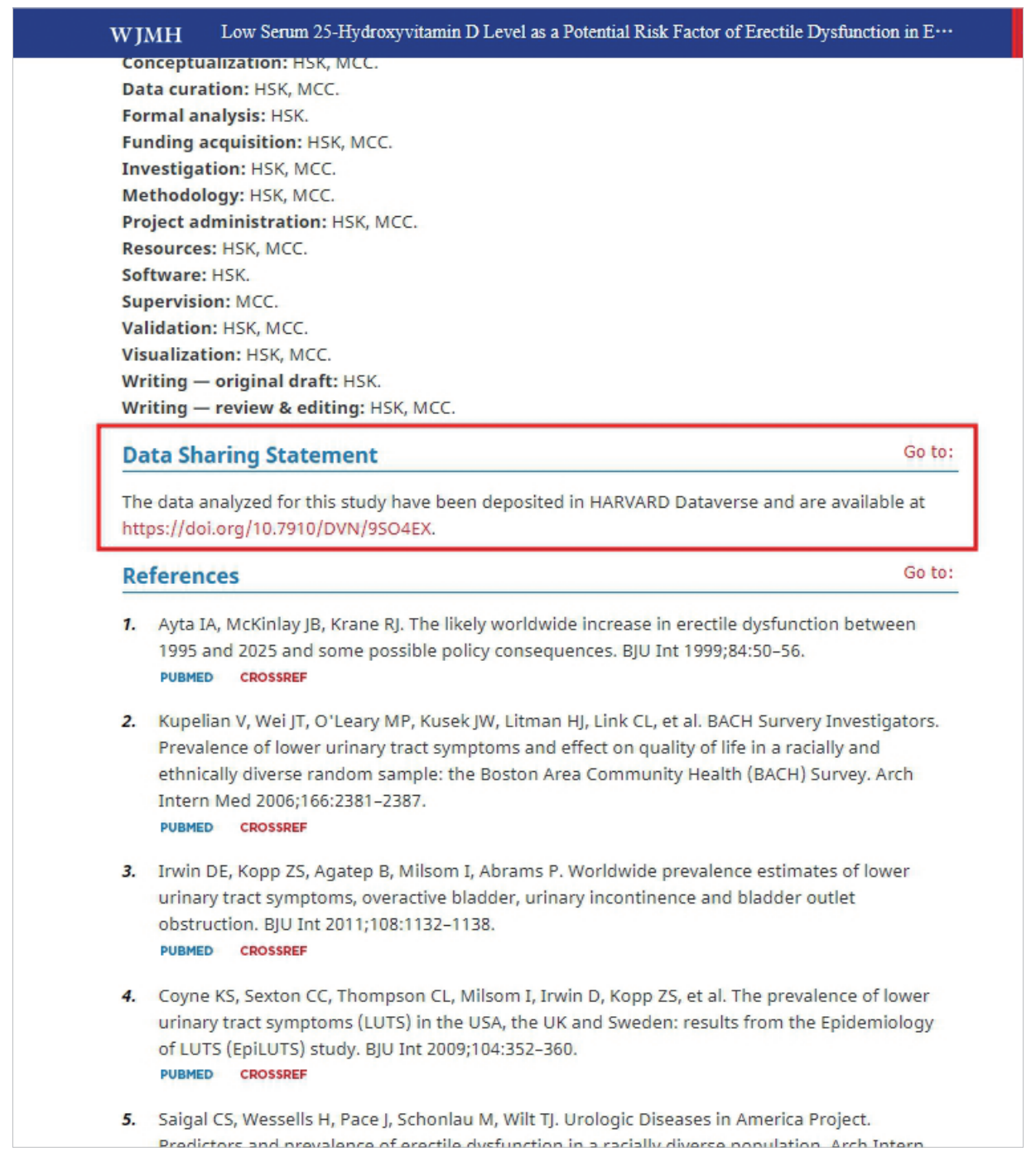

Fig. 5. Data sharing statement added to the manuscript note. 


\section{Conclusion}

For editors and publishers, sharing data can be a cumbersome process. However, data sharing provides opportunities for new research through the collaborative use of existing data. It may also increase the likelihood that the paper will be cited. The trend of data sharing is expected to spread widely across scientific and medical journals in all fields. Accordingly, platforms that provide storage and services for sharing data will gradually diversify. Therefore, in the field of scientific journal publication, it is recommended that editors select an appropriate platform and participate in the new trend of data sharing.

\section{Conflict of Interest}

No potential conflict of interest relevant to this article was reported.

\section{Funding}

The authors received no financial support for this article.

\section{References}

1. Taichman DB, Sahni P, Pinborg A, et al. Data sharing statements for clinical trials: a requirement of the International Committee of Medical Journal Editors. J Korean Med Sci 2017;32:1051-3. https://doi.org/10.3346/jkms.2017.32.7. 1051

2. Costanzo PR, Suarez SM, Kozak AE, Knoblovits P. Seasonal variations in sex steroids in a young male population and their relationship with plasma levels of Vitamin D. World J Mens Health 2021;39:e30. https://doi.org/10.5534/wjmh. 200156

3. Shirai M, Miyoshi Y, Ogasa T, et al. Oral testofen, L-citrulline, resveratrol, and caffeine supplement drink improves sexual function in men with phosphodiesterase 5 inhibitors: randomized, double-blind, placebo-controlled crossover pilot study. World J Mens Health 2021;39:733-9. https://doi. org/10.5534/wjmh.200129

4. Kim HS, Cho MC. Low serum 25-hydroxyvitamin D level as a potential risk factor of erectile dysfunction in elderly men with moderate to severe lower urinary tract symptoms. World J Mens Health 2022;40:139-48. https://doi.org/10. 5534/wjmh.200176 